\title{
Varus alignment of the proximal tibia is associated with structural progression in early to moderate varus osteoarthritis of the knee
}

\author{
Jonathan S. Palmer ${ }^{1}$ (D - Luke D. Jones ${ }^{2} \cdot$ A. Paul Monk ${ }^{3} \cdot$ Michael Nevitt $^{4} \cdot$ John Lynch $^{4} \cdot$ David J. Beard $^{5}$. \\ M. K. Javaid ${ }^{5}$. Andrew J. Price ${ }^{5}$
}

Received: 11 September 2019 / Accepted: 16 December 2019 / Published online: 21 January 2020

(c) The Author(s) 2020

\begin{abstract}
Purpose Lower limb malalignment is a strong predictor of progression in knee osteoarthritis. The purpose of this study is to identify the individual alignment variables that predict progression in early to moderate osteoarthritis of the knee.

Method A longitudinal cohort study using data from the Osteoarthritis Initiative. Intotal, 955 individuals (1329 knees) with early to moderate osteoarthritis (Kellgren-Lawrence grade 1,2 or 3) were identified. All subjects had full-limb radiographsanalysed using the Osteotomy module within Medicad $^{\circledR}$ Classic (Hectec GMBH) togive a series of individual alignment variables relevant to the coronal alignment of thelower limb. Logistic regression models, with generalised estimating equations wereused to identify which of these individual alignment variables predict symptomworsening (WOMAC score $>9$ points) and or structural progression (joint spacenarrowing progression in the medial compartment $>0.7 \mathrm{~mm}$ ) over 24 months. Results Individual alignment variable were associated with both valgus and varusalignment (mechanical Lateral Distal Femoral Angle, Medial Proximal Tibial Angle andmechanical Lateral Distal Tibial Angle). Only the Medial Proximal Tibial Angle wassignificantly associated with structural progression and none of the variables wasassociated with symptom progression. The odds of joint space narrowing progressionin the medial compartment occurring at 24 months increased by $21 \%$ for every onedegree decrease (more varus) in Medial Proximal Tibial Angle $(p<0.001)$

Conclusions Our results suggest that the risk of structural progression in the medialcompartment is associated with greater varus alignment of the proximal tibia.

Level of evidence Level III, retrospective cohort study.
\end{abstract}

Keywords Osteoarthritis $\cdot$ Knee $\cdot$ Mechanical alignment $\cdot$ Coronal $\cdot$ Proximal tibial angle $\cdot$ MPTA

Andrew J. Price

Andrew.price@ndorms.ox.ac.uk

1 Orthopaedic Department, Dorset County Hospital, Dorchester DT1 2JY, UK

2 Orthopaedic Department, Chelsea and Westminster Hospital, London SW10 9NH, UK

3 Auckland Bioengineering Institute, University of Auckland, Auckland 1142, New Zealand

4 San Francisco Coordinating Center, University of California, San Francisco, USA

5 Nuffield Department of Orthopaedics Rheumatology and Musculoskeletal Sciences, University of Oxford, Old Rd, Oxford OX3 7LD, UK

\section{Introduction}

Early to moderate knee osteoarthritis (OA) is common, hard to treat and can be debilitating for symptomatic individuals [13]. These patients are said to be in a "treatment gap" [14] where effective therapeutic interventions are limited.

A clear understanding of the predictors that cause structural progression, symptom worsening or non-progression more likely in individuals with symptomatic early to moderate knee OA is necessary.

Longitudinal cohort studies have confirmed that lower limb malalignment is a potent predictor of both incidence and progression of knee OA [9, 18, 20]. The term 'constitutional varus' has been used to describe the varus deformity seen in healthy individuals [2]. More recently, a wide variation in femoral and tibial alignment has been demonstrated in the healthy adult population [11]. Consequently, it has 
been suggested that traditional coronal alignment descriptions of neutral, varus and valgus using the hip-knee-ankle angle may oversimplify what is a highly variable characteristic with multiple possible phenotypes [10].

With this in mind, a longitudinal cohort study was designed using data from the Osteoarthritis Initiative (OAI). The aim was to identify whether the individual alignment variables that contribute to coronal alignment of the lower limb have a uniform effect on symptom and/or structural progression in symptomatic early to moderate knee OA. In other words, the study aimed to determine whether traditional alignment descriptions of varus, valgus and neutral are sufficient to identify patients with knee OA who are at risk of progression or is a more detailed description of coronal alignment required.

Our hypothesis was that traditional alignment descriptions oversimplify coronal alignment and that the individual alignment variables contributing to coronal alignment will have varying effects on progression of knee OA.

\section{Material and methods}

\section{Study sample}

The study population comprised participants from the OAI. The OAI is a multi-centre, longitudinal, observational cohort study focusing primarily on the natural history of knee OA [15].

Eligible subjects had at least one knee with Kellgren-Lawrence (KL) grade 1, 2 or 3 and symptoms in the same knee at recruitment. Symptoms were defined as knee pain, aching or stiffness: more than half the days of a month in the past year in the same knee. If a subject had two eligible knees, then both knees were enrolled in the study. To be eligible for the study, both clinical and radiographic outcomes at 24 months had to be available. All individuals had to have a full-limb radiograph (FLR) with all landmarks clearly visible and available for analysis.

The OAI did not perform FLRs on any individuals at recruitment. Individuals with established OA (KL grade 2 or more and symptoms) had an FLR at 12 months. FLRs were acquired after 12 months in the remaining cohort including those subjects with KL grade 1 at recruitment (see Fig. 1).

Subjects with a significant fixed flexion deformity $\left(\mathrm{FFD}>10^{\circ}\right)$ at recruitment were excluded, as a small degree of rotation in such individuals can significantly affect the accuracy of coronal alignment measurements [19]. As no FLRs were taken at recruitment, some subjects demonstrated progression of OA on their FLR such that bony attrition was present on either the tibial plateau or femoral condyle. These subjects were excluded as such intra-articular deformity may have exaggerated

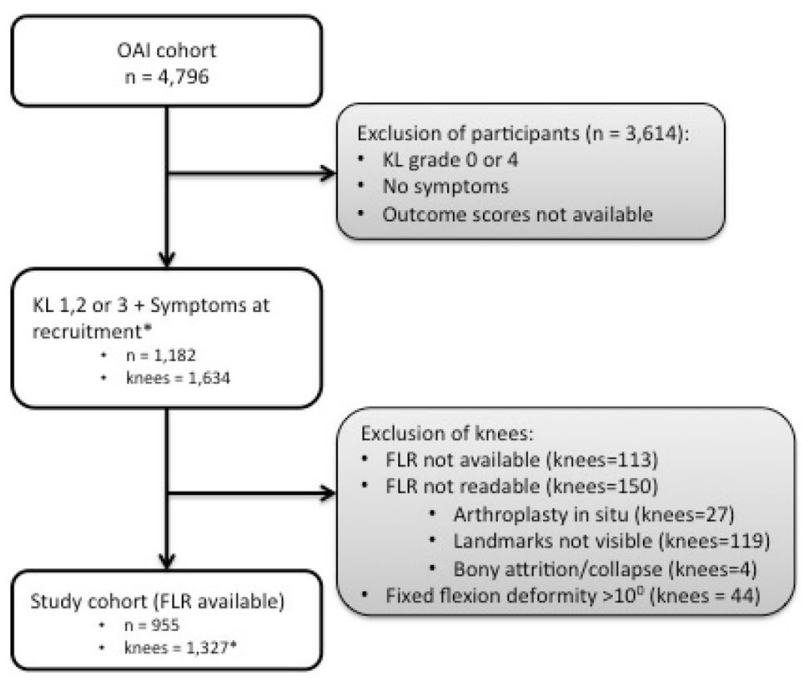

Fig. 1 Formation of the study cohort. Flowchart illustrates the formation of the study cohort. *Timepoint at which FLRs were taken; 12 months $(n=965), 24$ months $(n=230), 36$ months $(n=112)$, 48 months $(n=20)$

Table 1 Characteristics of the study cohort

\begin{tabular}{ll}
\hline Characteristic & Study cohort $(n=955)$ \\
\hline Age (mean; SD) & $60.7( \pm 8.9)$ \\
Sex (\% female) & $57 \%$ \\
BMI $\left(\mathrm{kg} / \mathrm{m}^{2} ;\right.$ mean) & 29.9 \\
Race (\% Caucasian) & $69.8 \%$ \\
Co-morbidities (\% none) & $70.5 \%$ \\
Smoker $(\%)$ & $7.2 \%$ \\
KL grade $(\%)$ & $1=17 \%$ \\
& $2=50 \%$ \\
& $3=33 \%$ \\
\hline
\end{tabular}

any malalignment. Similarly, subjects were also excluded if a total hip replacement or total knee replacement was present on the FLR, as this would have given erroneous measurements for coronal alignment. Subjects who were unable to progress as their joint space was already too narrow at baseline $(<1 \mathrm{~mm})$ were excluded from the study, as their disease was too severe to be considered early to moderate knee OA.

Information regarding the acquisition of LLRs within OAI is publicly available [15]. Weight-bearing FLRs were taken of both lower limbs simultaneously with toes perpendicular to the film, the femoral epicondyles were kept parallel to the cassette and knees were kept fully extended whilst distributing the participants' weight evenly.

A flow chart outlining the selection of the cohort is given in Fig. 1 and the baseline demographic details of the cohort are outlined in Table 1. 


\section{Outcomes}

Symptom progression was defined as an increase in WOMAC score (symptom worsening) of $>9$ points at 24 months compared to baseline [1]. Subjects with severe symptoms at baseline (WOMAC $>91$ points) who could not progress by more than 9 points were defined as progressing if their symptoms were sustained (WOMAC $>91$ points) at 24 months. WOMAC scores were normalized to have a $0-100$ range.

Structural progression was defined as joint space narrowing (JSN) progression in the medial compartment $(>0.7 \mathrm{~mm})[8]$.

\section{Radiographic assessment}

All available FLRs were viewed as Digital Imaging and Communications in Medicine (DICOM) files and analysed using the Osteotomy Module within Medicaid ${ }^{\circledR}$ Classic (Hectec GMBH) a surgical planning tool with high interrater reliability [17]. It generates mechanical alignment measurements including weight-bearing axis (WBA), mechanical lateral proximal femoral angle (mLPFA), mechanical lateral distal femoral angle (mLDFA), medial proximal tibial angle (MPTA), mechanical lateral distal tibial angle (mLDTA) and medial joint line convergence angle (JLCA_med) (Fig. 2).

\section{Observer reliability}

A single observer (JP) reviewed all the FLRs in the cohort. Intraclass correlation coefficients (ICCs) were performed on readings of the first 20 limbs performed by the same observer (JP) after a 6-week interval (intra-observer reliability) and by another orthopaedic registrar trained in the use of Medicaid ${ }^{\circledR}$ Classic (Hectec GMBH) (inter-observer reliability). ICCs were then repeated for readings of these 20 limbs made after the first 100, 200 and 500 LLR readings to identify any observer drift (JP).

Excellent intra-observer reliability was demonstrated with intraclass correlation coefficients $>0.90$ for all alignment variables measured at baseline compared to those measured after the first 100 readings. There was no evidence of any observer drift with ICCs being maintained at $>0.80$ after the first 200 and first 500 readings were performed. Excellent inter-observer reliability was also demonstrated $(>0.80)$ across all readings.

\section{Statistical analysis}

\section{Alignment variables by gender and baseline KL grade}

Differences in alignment variables (mLPFA, mLDFA, MPTA, mLDTA) by gender and baseline KL grade were determined using generalised estimating equations (GEEs).

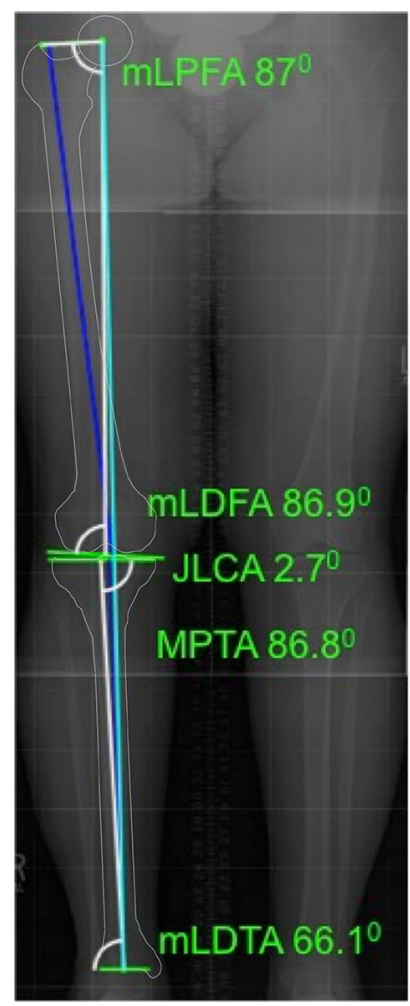

Fig. 2 Alignment variables were obtained using Medicaid ${ }^{\circledR}$ Classic $($ Hectec GMBH) for all individuals within the cohort. Mechanical alignment measurements included weight-bearing axis (WBA), mechanical lateral proximal femoral angle (mLPFA), mechanical lateral distal femoral angle (mLDFA), medial proximal tibial angle (MPTA), mechanical lateral distal tibial angle (mLDTA) and joint line convergence angle (JLCA). The light blue line indicates the weight-bearing axis expressed as a percentage of the medial-lateral tibial plateau (35.3\% in this plan). NB: the outline of the femur and tibia has been highlighted in white to aid interpretation of this figure

\section{Alignment variables that predict overall alignment (e.g. valgus or varus)}

The hip-knee-ankle angle (HKA) was converted to a binary outcome to separate those with valgus alignment $\left(\right.$ HKA $\left.>0^{\circ}\right)$ and those with varus alignment $\left(\right.$ HKA $\left.<0^{\circ}\right)$ [18]. Logistic regression models with GEEs were used to account for the potential correlation of observations that may arise from knees belonging to the same individual. The GEEs were used to identify which of the alignment variables (mLPFA, mLDFA, MPTA, mLDTA) predicted whether a subject was in varus or valgus alignment. Standardised coefficients were calculated to enable an assessment of how many standard deviations the HKA changed, per standard deviation increase in the alignment variable. In doing so, it was possible to determine which of the individual alignment variables, if any, had the greatest effect on the HKA. 


\section{Alignment variables and their effect on progression}

Logistic regression models, with GEEs, were used to identify the baseline variables that predict symptom or structural progression at 24 months. Variables of interest were selected a priori: age, gender, BMI, number of co-morbidities, smoking status, race, employment status, and previous history of knee surgery. In addition, the individual mechanical alignment measurements were included in the statistical model: WBA, mLPFA, mLDFA, MPTA, mLDTA (Fig. 2).

Adjustments were made to some variables to facilitate their incorporation into the statistical models and subsequent interpretation. Employment status was converted from a categorical variable with four possible outcomes into a binary outcome (paid work vs not in paid work). Smoking status was converted from a categorical variable with four possible outcomes to a binary outcome (any smoking history vs no smoking history). The majority of participants in the OAI cohort reported their ethnic origin as black or Caucasian $(97.1 \%)$. For this reason, the variable for race was simplified to a binary outcome (Caucasian vs non-Caucasian).

Each variable was added to the model in turn to assess its direct effect on the outcome of interest. Multivariate analysis, with all variables included, was then performed to explore and quantify the influence of these variables on OA progression. All models were adjusted for baseline JSN and baseline WOMAC score. Relationships between predictor variables and outcome were expressed as odds ratios (OR) and a $p$ value $<0.05$ was considered statistically significant. The independent variables included in the model were tested for collinearity using the variance inflation factor (VIF).

\section{Multinomial regression analysis}

Outcome groups were determined based on symptomatic worsening (WOMAC $>9$ points) [1] and structural progression (JSN $>0.7 \mathrm{~mm}$ ) [8]. Multinomial regression was then performed to determine the effect of predictor variables on the likelihood of an individual progressing to one of three outcome groups compared to the likelihood of progressing to a fourth referent group:

1. Non-progressor-no symptom worsening and no structural progression at 24 months (referent group).

2. Structural progressor-no symptom worsening but structural progression at 24 months.

3. Symptom progressor-symptom worsening but no structural progression at 24 months.

4. Structure and symptom progressor-symptom worsening and structural progression at 24 months.

Results were expressed as relative risk ratio (RRR) and $p$ values of $<0.05$ were considered statistically significant.
Table 2 The difference in alignment variables by gender is summarised

\begin{tabular}{lccccc}
\hline & \multicolumn{2}{l}{ Males $(n=535)$} & & \multicolumn{2}{c}{ Females $(n=792)$} \\
\cline { 2 - 3 } \cline { 5 - 6 } & Mean & SD & & Mean & SD \\
\hline mLPFA & $91.8^{\circ}$ & 5.4 & & $92.0^{\circ}$ & 5.8 \\
mLDFA* & $87.5^{\circ}$ & 2.0 & & $87.3^{\circ}$ & 2.4 \\
MPTA* $^{*}$ & $86.6^{\circ}$ & 2.3 & & $87.9^{\circ}$ & 2.4 \\
mLDTA* $_{\text {JLCA_med }}$ & $86.2^{\circ}$ & 4.0 & & $87.3^{\circ}$ & 3.5 \\
\hline
\end{tabular}

*Indicates a significant difference by gender $(p<0.05)$
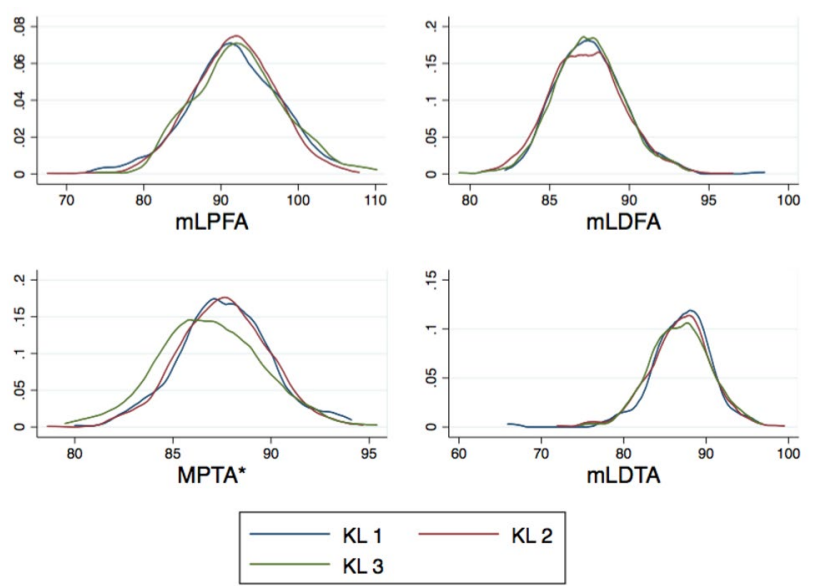

Fig. 3 Kernel density plot graphs illustrating the differences in alignment variables observed by baseline KL grade. ${ }^{*} p \leq 0.05$

All statistical analyses were performed using Stata 13.1 (StataCorp. 2013. Stata Statistical Software: Release 13. College Station, TX: StataCorp LP).

\section{Results}

955 individuals with 1,327 knees were included in the study.

Differences in alignment variables by gender and baseline KL grade are outlined in Table 2 and Fig. 3, respectively.

Standardised coefficients revealed that the scale of effect on varus and valgus alignment was similar for both MPTA and mLDFA (see Table 3).

No alignment variables predicted symptom worsening at 24 months. Predictors of symptom worsening (WOMAC $>9$ points) at 24 months included WOMAC score $(p<0.001)$ and BMI $(p=0.014)$ at recruitment. For every one unit increase in baseline BMI, the odds of developing worsening knee pain at 24 months increased by $6 \%$. For every one unit increase in baseline WOMAC, the odds of developing worsening knee pain at 24 months decreased by $3 \%$ (see Table 4). 
Table 3 The influence of alignment variables on overall alignment is summarised

\begin{tabular}{llllcc}
\hline Alignment & Predictor & Odds ratio & $95 \%$ CI & $p$ value & $\begin{array}{l}\text { Stand- } \\
\text { ardised } \\
\text { coeffi- } \\
\text { cient }\end{array}$ \\
\hline Varus & mLPFA & 1.03 & $0.99-1.08$ & 0.15 & 0.02 \\
& mLDFA* $^{*}$ & 3.63 & $2.57-5.12$ & $<0.001$ & 0.58 \\
& MPTA* & 0.23 & $0.16-0.34$ & $<0.001$ & -0.60 \\
Valgus & mLDTA* $^{*}$ & 1.11 & $1.04-1.19$ & 0.003 & 0.03 \\
& mLPFA & 0.97 & $0.93-1.02$ & 0.20 & -0.005 \\
& mLDFA* & 0.27 & $0.19-0.39$ & $<0.001$ & -0.58 \\
& MPTA* & 4.44 & $2.91-6.77$ & $<0.001$ & 0.61 \\
& mLDTA* & 0.90 & $0.84-0.97$ & 0.005 & -0.03 \\
\hline
\end{tabular}

$* p \leq 0.05$

The only variable that predicted structural progression at 24 months (JSN $>0.7 \mathrm{~mm}$ ) was MPTA. The odds of JSN progression occurring at 24 months increased by $21 \%$ for every one degree decrease (more varus) in MPTA $(p<0.001)$ (see Table 4).

\section{Multinomial regression analysis}

The cohort was split based on the four possible outcome groups. The majority of individuals were in the no-progression group (69.1\%). This group was the referent group from which the RRR of a subject falling into one of the remaining three groups was calculated.
Only baseline WOMAC had a significant effect on the risk of being in the symptom worsening group $(n=158)$ relative to the referent group. For every one unit increase in the baseline WOMAC, the relative risk of being in the symptom worsening group decreased by a factor of $0.98(p=0.001)$.

Baseline WOMAC and MPTA had significant effects on the risk of being in the structural progression group $(n=195)$ relative to the referent group. For every one degree decrease in the MPTA (more varus), the relative risk of being in the structural progression group increased by a factor of 1.16 $(p<0.001)$. For every unit increase in the baseline WOMAC, the relative risk of being in the structural progression group increased by a factor of $1.01(p=0.02)$.

Baseline WOMAC, MPTA and gender had significant effects on the risk of being in the symptom and structural progression group $(n=57)$ relative to the referent group. For every one degree decrease in the MPTA (more varus), the relative risk of being in the symptom and structural progression group increased by a factor of $1.16(p=0.03)$. For every unit increase in the baseline WOMAC, the relative risk of being in the symptom and structural progression group decreased by a factor of $0.97(p=0.005)$. For female participants, the relative risk of being in the symptom and structural progression group, relative to the referent group, is expected to increase by a factor of $2.7(p=0.01)$.

\section{Collinearity}

None of the variables in the multivariate model had a VIF greater than two and the mean of the VIF scores was close to
Table 4 Multivariate regression analysis to determine the influence of all baseline variables on symptom worsening (WOMAC $>9$ points) and structural progression (JSN $>0.7 \mathrm{~mm}$ ) over 24 months

\begin{tabular}{|c|c|c|c|c|c|c|}
\hline \multirow[t]{2}{*}{ Variable of interest } & \multicolumn{3}{|c|}{$\begin{array}{l}\text { Symptom worsening (WOMAC }>9 \\
\text { points) }\end{array}$} & \multicolumn{3}{|c|}{ Structural progression $(\mathrm{JSN}>0.7 \mathrm{~mm})$} \\
\hline & Odds ratio & $95 \% \mathrm{CI}$ & $p$ value & Odds ratio & $95 \% \mathrm{CI}$ & $p$ value \\
\hline Baseline WOMAC* & 0.97 & $0.95-0.98$ & $<0.001$ & 1.01 & $1.00-1.02$ & 0.16 \\
\hline Baseline JSN & 0.95 & $0.80-1.13$ & 0.53 & 1.06 & $0.89-1.27$ & 0.50 \\
\hline Age & 0.99 & $0.96-1.02$ & 0.41 & 1.01 & $0.98-1.04$ & 0.56 \\
\hline Gender & 1.46 & $0.90-2.37$ & 0.13 & 1.18 & $0.70-1.98$ & 0.53 \\
\hline BMI* & 1.06 & $1.01-1.11$ & 0.01 & 1.02 & $0.97-1.07$ & 0.39 \\
\hline Co-morbidities & 1.10 & $0.90-1.40$ & 0.45 & 0.89 & $0.66-1.16$ & 0.36 \\
\hline Race & 0.76 & $0.47-1.24$ & 0.27 & 1.15 & $0.67-1.96$ & 0.62 \\
\hline Employment & 0.63 & $0.38-1.05$ & 0.07 & 1.22 & $0.70-2.12$ & 0.49 \\
\hline Smoker & 1.25 & $0.82-1.92$ & 0.30 & 1.30 & $0.82-2.06$ & 0.27 \\
\hline Previous knee surgery & 1.19 & $0.70-2.00$ & 0.52 & 0.97 & $0.56-1.69$ & 0.91 \\
\hline mLPFA & 1.03 & $0.99-1.06$ & 0.18 & 1.02 & $0.98-1.06$ & 0.39 \\
\hline mLDFA & 0.92 & $0.84-1.01$ & 0.09 & 1.05 & $0.95-1.16$ & 0.35 \\
\hline MPTA** & 1.05 & $0.96-1.15$ & 0.31 & 0.79 & $0.71-0.88$ & $<0.001$ \\
\hline mLDTA & 1.02 & $0.96-1.08$ & 0.59 & 1.02 & $0.96-1.08$ & 0.50 \\
\hline
\end{tabular}

*Indicates significant predictor of symptom worsening at 24 months $(p<0.05)$

**Indicates significant predictor of structural progression at 24 months $(p<0.05)$ 
one representing inconsequential collinearity for the model [16].

\section{Further analysis}

Higher baseline KL grade was associated with a more varus MPTA. Baseline KL grade was not included in the statistical model, as it has a significant correlation with baseline JSN (Pearson correlation $=-0.4 ; p \leq 0.001$ ). To ensure that the predictive effects of MPTA are not the result of an unseen interaction between MPTA and baseline KL grade, the models were repeated with baseline KL grade exchanged for baseline JSN in the multivariate models for progression. The predictors of structural and symptom progression seen in the above results sections remained stable following this adjustment.

\section{Discussion}

The most important finding of this study was that a varus MPTA was associated with significant structural progression in subjects with medial compartment knee OA. The odds of structural progression occurring at 24 months increased by $21 \%$ for every one degree decrease (more varus) in MPTA $(p<0.001)$. Whilst other factors contribute to both valgus and varus alignment (mLDFA, MPTA and mLDTA), they do not have a significant effect on structural progression. Subsequent, multinomial regression identified that for every one degree decrease in MPTA, the relative risk of being in the symptom and structural progression group and the structural progression group, relative to the referent group, increased by a factor of 1.16 .

Several studies have reported on varus malalignment and its potent effects on structural progression in knee OA $[6,18]$. Our study demonstrates that traditional alignment descriptions of varus, valgus and neutral are too simplistic and a more detailed assessment of coronal alignment is required. Hirschmann et al. have described functional knee phenotypes that acknowledge individual variations in alignment of the proximal tibia and distal femur and recommend that such phenotypes be used to facilitate an individualised approach to TKA surgery [12]. Our study highlights the importance of these individual alignment variables and their influence on knee OA progression.

This study challenges the observations made by other groups. Cooke et al. compared the alignment of 127 patients with symptomatic varus $\mathrm{OA}$ to 75 varus aligned healthy individuals [7]. Significant differences were observed in distal femoral geometry between the two groups leading the authors to conclude that it was distal femoral alignment that drove the development of medial compartment OA. This cross-sectional study did not have follow-up data from which to confirm this finding and it is not supported by our study.

No association was made between MPTA and symptom worsening at 24 months. A follow-up of 24 months may have been insufficient to detect any effect of individual alignment variables on symptom worsening at 24 months. A high baseline WOMAC score was, however, associated with reduced odds of symptom worsening at 24 months. Subjects for our cohort were selected based on their experiences of pain over the preceding 12 months, as such baseline WOMAC scores are likely to reflect an extreme measurement in terms of knee pain and subsequent regression to the mean [4] as time progresses.

Our study has other noteworthy limitations. The exclusion of certain variables could be a potential source of bias. Subjects with a FFD of more than 10 degrees were excluded from the study. A high FFD may in itself be associated with progression and by excluding this variable we may have biased the sample towards a risk group that is not related to FFD. The exclusion of those subjects who had evidence of bony attrition $(n=4)$ or arthroplasty surgery $(n=27)$ on their FLR may have selected out some important individuals with rapidly progressive $\mathrm{OA}$. This reflected the fact that the OAI cohort did not have FLRs performed until at least 12 months after recruitment. As such, a participant may have progressed to end-stage disease before any assessment of coronal alignment was possible.

The participants in this study were selected to reflect patients who find themselves in the treatment gap. The average age of the cohort was 60.7 years and the mean MPTA was $87.4^{\circ}( \pm 2.4)$, similar to that reported in a healthy population described by Bellemans et al. [87.04 ${ }^{\circ}( \pm 2.07)$ ] [2]. Younger age patients $(<45$ years) are not represented in this study and whilst some severe varus deformity will be present, the cohort as a whole shows very little variation in alignment. By contrast, a prospective study of individuals undergoing high tibial osteotomy with an average age of 47.5 years had a mean proximal tibial angle of $81.44^{\circ}$ $( \pm 4.51)$ [3]. Rates of progression in these younger patients with more severe proximal tibia vara would be of interest and has not been fully delineated.

Our cohort included subjects with symptomatic mild to moderate knee OA (KL grade 1, 2 and 3). This heterogeneous group was chosen as they reflect patients with symptomatic knee osteoarthritis and structural changes related to osteoarthritis that are not generally considered suitable for knee replacement surgery. Identifying risk factors for progression in this group are of interest as they have implications for surgical intervention.

Despite these limitations, this study has many strengths. The study population is large $(n=955 ; \mathrm{kn}=1327)$ and the statistical analysis was established a priori. 
Proximal tibial alignment has clinical relevance. In the context of varus deformity, the proximal tibia can be corrected with a surgical intervention such as an osteotomy. Bonnin and Chambat reported superior clinical outcomes for patients undergoing osteotomy for medial OA for patients with a constitutional varus deformity of the proximal tibia [5]. This study supports the use of a high tibial osteotomy in the presence of proximal tibia vara, as it is this deformity that is driving structural progression over 24 months.

\section{Conclusion}

This study has confirmed that whilst malalignment is a significant predictor of structural progression in symptomatic early knee $\mathrm{OA}$, it is specifically the alignment of the proximal tibia that is associated with this phenomenon. Traditional alignment descriptions using the hip-knee-angle oversimplify coronal alignment. Future studies that aim to observe the effect of an intervention on structural progression of knee osteoarthritis will need to consider the effect of these individual alignment variables.

Acknowledgements The first author's studies were funded in part by the National Institute for Health Research, Biomedical Research Unit, Oxford. The views expressed in this publication are those of the authors and not necessarily those of the MRC, NHS, NIHR or the Department of Health.

Author contributions JP contributed to all aspects of the study including study design, research methodology, data collection, statistical analysis and write-up. LDJ contributed to creation of de novo OAI cohort, study design and data collection. APM contributed to study design, statistical analysis and collection of data. MN contributed to the study design, write-up and development of cohort. JL contributed to study design and development of cohort. DJB contributed to research methodology, synthesis of data and write-up. KJ contributed to study design, research methodology, statistical analysis, data interpretation and write-up. AJP contributed to study design, direction of study, synthesis of data and write-up.

Funding The first author's studies were funded in part by the National Institute for Health Research, Biomedical Research Unit, Oxford. The views expressed in this publication are those of the authors and not necessarily those of the MRC, NHS, NIHR or the Department of Health. MKJ was supported by the National Institute for Health Research (NIHR) Oxford Biomedical Research Centre (BRC). The views expressed are those of the author(s) and not necessarily those of the NHS, the NIHR or the Department of Health. The OAI is a public-private partnership comprised of five contracts (N01-AR-2-2258; N01-AR-2-2259; N01-AR-2-2260; N01-AR-2-2261; N01-AR-2-2262) funded by the National Institutes of Health, a branch of the Department of Health and Human Services, and conducted by the OAI Study Investigators. Private funding partners include Merck Research Laboratories; Novartis Pharmaceuticals Corporation, GlaxoSmithKline; and Pfizer, Inc. Private sector funding for the OAI is managed by the Foundation for the National Institutes of Health. This manuscript was prepared using an OAI public use data set and does not necessarily reflect the opinions or views of the OAI investigators, the $\mathrm{NIH}$, or the private funding partners.

\section{Compliance with ethical standards}

Conflict of interest There are no conflicts of interests as outlined by the signed statements sent from each of the authors.

Ethical approval The data was from a publicy availale database (the OsteoArthritis Initiative) from the U.S. As such ethical approval was not required.

Open Access This article is licensed under a Creative Commons Attribution 4.0 International License, which permits use, sharing, adaptation, distribution and reproduction in any medium or format, as long as you give appropriate credit to the original author(s) and the source, provide a link to the Creative Commons licence, and indicate if changes were made. The images or other third party material in this article are included in the article's Creative Commons licence, unless indicated otherwise in a credit line to the material. If material is not included in the article's Creative Commons licence and your intended use is not permitted by statutory regulation or exceeds the permitted use, you will need to obtain permission directly from the copyright holder. To view a copy of this licence, visit http://creativecommons.org/licenses/by/4.0/.

\section{References}

1. Angst F, Aeschlimann A, Michel BA, Stucki G (2002) Minimal clinically important rehabilitation effects in patients with osteoarthritis of the lower extermities. J Rheumatol 25:131-138

2. Bellemans J, Colyn W, Vandenneucker H, Victor J (2012) The Chitranjan Ranawat award: Is neutral mechanical alignment normal for all patients? The concept of constitutional varus. Clin Orthop Relat Res 470:45-53

3. Birmingham TB, Giffin JR, Chesworth BM, Bryant DM, Litchfield RB, Willits K, Jenkyn TR, Fowler PJ (2009) Medial opening wedge high tibial osteotomy: a prospective cohort study of gait, radiographic, and patient-reported outcomes. Arthritis Rheum 61:648-657

4. Bland JM, Altman DG (1994) Statistics notes: some examples of regression towards the mean. BMJ 309:780-780

5. Bonnin M, Chambat $P$ (2004) Current status of valgus angle, tibial head closing wedge osteotomy in media gonarthrosis. Orthopade 33:135-142

6. Cerejo R, Dunlop DD, Cahue S, Channin D, Song J, Sharma L (2002) The influence of alignment on risk of knee osteoarthritis progression according to baseline stage of disease. Arthritis Rheum 46:2632-2636

7. Cooke D, Scudamore A, Li J, Wyss U, Bryant T, Costigan P (1997) Axial lower limb alignment. Comparison of knee geometry in normal volunteers and osteoarthritis patients. Osteoarthritis CartilageCartilage 5:39-47

8. Eckstein F, Collins JE, Nevitt MC, Lynch JA, Kraus VB, Katz JN, Losina E, Wirth W, Guermazi A, Roemer FW, Hunter DJ, Oa F, Consortium B (2015) Cartilage thickness change as an imaging biomarker of knee osteoarthritis progression: data from the foundation for the national institutes of health osteoarthritis biomarkers consortium. Arthritis Rheumatol 67:3184-3189

9. Hayashi D, Englund M, Roemer FW, Niu J, Sharma L, Felson DT, Crema MD, Marra MD, Segal N, Lewis CE, Nevitt MC, Guermazi A (2012) Knee malalignment is associated with an increased risk for incident and enlarging bone marrow lesions in the more loaded compartments: the MOST study. Osteoarthr Cartil 20:1227-1233 
10. Hirschmann MT, Hess S, Behrend H, Amsler F, Leclercq V, Moser LB (2019) Phenotyping of hip-knee-ankle angle in young nonosteoarthritic knees provides better understanding of native alignment variability. Knee Surg Sports Traumatol Arthrosc Springer $27: 1378-1384$

11. Hirschmann MT, Moser LB, Amsler F, Behrend H, Leclercq V, Hess S (2019) Phenotyping the knee in young non-osteoarthritic knees shows a wide distribution of femoral and tibial coronal alignment. Knee Surg Sports Traumatol Arthrosc 27:1385-1393

12. Hirschmann MT, Moser LB, Amsler F, Behrend H, Leclerq V, Hess S (2019) Functional knee phenotypes: a novel classification for phenotyping the coronal lower limb alignment based on the native alignment in young non-osteoarthritic patients. Knee Surg Sports Traumatol Arthrosc 27:1394-1402

13. Jones LD, Bottomley N, Harris K, Jackson W, Price AJ, Beard DJ (2014) The clinical symptom profile of early radiographic knee arthritis: a pain and function comparison with advanced disease. Knee Surg Sports Traumatol Arthrosc 24:161-168

14. London NJ, Miller LE, Block JE (2011) Clinical and economic consequences of the treatment gap in knee osteoarthritis management. Med Hypotheses 76:887-892

15. Nevitt M, Felson D, Lester G (2006) The osteoarthritis initiative: protocol for the cohort study. Available via OAI:Home. https://oai. epi-ucsf.org/datarelease. Accessed 25 Mar 2018.
16. O'Brien RM (2007) A caution regarding rules of thumb for variance inflation factors. Qual Quant 41:673-690

17. Schröter S, Ihle C, Mueller J, Lobenhoffer P, Stöckle U, Heerwaarden R (2012) Digital planning of high tibial osteotomy. Interrater reliability by using two different software. Knee Surg Sports Traumatol Arthrosc 21:189-196

18. Sharma L, Song J, Felson DT, Cahue S, Shamiyeh E, Dunlop DD (2001) The role of knee alignment in disease progression and functional decline in knee osteoarthritis. JAMA 286:188-195

19. Shetty GM, Mullaji A, Khalifa AA, Ray A, Nikumbha V (2017) The effect of sagittal knee deformity on preoperative measurement of coronal mechanical alignment during total knee arthroplasty. Knee Surg Relat Res 29:110-114

20. Tanamas S, Hanna FS, Cicuttini FM, Wluka AE, Berry P, Urquhart DM (2009) Does knee malalignment increase the risk of development and progression of knee osteoarthritis? A systematic review. Arthritis Rheum 61:459-467

Publisher's Note Springer Nature remains neutral with regard to jurisdictional claims in published maps and institutional affiliations. 